\title{
Groupoids and the algebra of rewriting in group presentations
}

\author{
N. D. Gilbert ${ }^{1}$ (D) E. A. McDougall ${ }^{1}$
}

Received: 21 October 2019 / Accepted: 7 September 2020 / Published online: 15 September 2020 (c) The Author(s) 2020

\begin{abstract}
Presentations of groups by rewriting systems (that is, by monoid presentations), have been fruitfully studied by encoding the rewriting system in a 2-complex-the Squier complex — whose fundamental groupoid then describes the derivation of consequences of the rewrite rules. We describe a reduced form of the Squier complex, investigate the structure of its fundamental groupoid, and show that key properties of the presentation are still encoded in the reduced form.
\end{abstract}

Keywords Presentation · Groupoid · Crossed module

Mathematics Subject Classification 20F05 $\cdot$ 20J05 $\cdot$ 20L05

\section{Introduction}

The study of the relationships between presentations of semigroups, monoids, and groups, and systems of rewriting rules has drawn together concepts from group and semigroup theory, low-dimensional topology, and theoretical computer science. Squier (1987) addressed the question of whether a finitely presented monoid with solvable word problem is necessarily presented by a finite, complete, string rewriting system. He proved that a monoid presented by a finite, complete, string rewriting system must satisfy the homological finiteness condition $\mathrm{FP}_{3}$ : indeed, an earlier result of Anick (1986) implies that such a monoid satisfies the stronger condition $F P_{\infty}$. These ideas are concisely surveyed in Cohen (1993), and more extensively in Otto and Kobayashi (1997). Since examples are known of finitely presented monoids with solvable word problem that do not satisfy $\mathrm{FP}_{3}$, Squier's work shows that such monoids need not be presented by finite, complete, string rewriting systems.

$凶 \quad$ N. D. Gilbert

N.D.Gilbert@hw.ac.uk

1 Department of Mathematics and The Maxwell Institute for the Mathematical Sciences, Heriot-Watt University, Edinburgh EH14 4AS, UK 
Squier et al. (1994) studied finite, complete, string rewriting systems for monoids and proved that the existence of such a system presenting a monoid $M$ implies a homotopical property-finite derivation type-defined for a graph that encodes the rewriting system. Moreover, they show that having finite derivation type does not depend on the particular rewriting system used to present $M$, and so is a property of $M$ itself and a necessary condition that $M$ should be presented by a finite, complete string rewriting system.

Finite derivation type is naturally thought of as a property of a 2-complex, the Squier complex associated to a monoid presentation $\mathcal{P}$, and obtained by adjoining certain 2-cells to the graph of Squier et al. (1994). This point of view was introduced independently by Pride (1995) and Kilibarda (1997), and then extensively developed in Guba and Sapir $(1997,2006)$ in terms of both string-rewriting systems, and more geometrically, in terms of directed 2-complexes. The theory developed by Kilibarda and then by Guba and Sapir focusses on the properties of diagram groups, which are fundamental groups of the Squier complex.

Kilibarda (1997) studied the fundamental groupoid of the Squier complex associated to a monoid presentation $[X: R]$. Gilbert (1998) showed that the fundamental groupoid is a monoid in the category of groupoids, and used this enriched structure to explain Pride's corresponding theory of diagram groups for monoid presentations of groups (Pride 1999).

The approach of Pride (1999) is based upon the addition of extra 2-cells to a Squier complex so as to realise a homotopy relation introduced in Cremanns and Otto (1996). This augmented Squier complex was called the Pride complex in Gilbert (1998) and denoted by $K^{+}$. Beginning with a group presentation $\mathcal{P}=\langle X: R\rangle$ of a group $G$, we obtain a monoid presentation of $G$ by adding relations $x x^{-1}=1=x^{-1} x$ for each $x \in X$, and the additional 2-cells correspond to possible overlaps in the use of such relations in the free reduction of words on $A=X \cup X^{-1}$. The outcome is that if $u$ and $v$ are freely equivalent then any two edge-paths in $K^{+}$from $u$ to $v$ that record this free equivalence are fixed-end-point homotopic, as required for a homotopy relation as defined in Cremanns and Otto (1996). Gilbert (1998) investigated the structure of the fundamental groupoid $\pi\left(K^{+}, A^{*}\right)$ and showed that there is a retraction map $\pi\left(K^{+}, A^{*}\right) \rightarrow \pi\left(K^{+}, F(X)\right)$ to the fundamental groupoid with vertex set the free group $F(X)$.

In this paper-which reconfigures the approach to monoid presentations of groups in Cremanns and Otto (1996) and is a somewhat belated sequel to Gilbert (1998) and Pride (1999)—we adopt a similar approach, but use a different modification of the Squier complex, defining the reduced Squier complex $\operatorname{Sq}^{\rho}(\mathcal{P})$ of a group presentation $\mathcal{P}=\langle X: R\rangle$ as a 2-complex having vertex set $F(X)$. We can then work directly with the fundamental groupoid $\pi\left(\mathrm{Sq}^{\rho}(\mathcal{P}), F(X)\right)$ and so avoid some of the technicalities from Gilbert (1998). In particular, we show that the set $\operatorname{star}_{1}\left(\operatorname{Sq}^{\rho}(\mathcal{P})\right)$ of homotopy classes of paths in $\pi\left(\mathrm{Sq}^{\rho}(\mathcal{P}), F(X)\right)$ that begin at $1 \in F(X)$ has a natural group structure, and the end-of-path map

$$
\mathbf{r}: \operatorname{star}_{1}\left(\mathrm{Sq}^{\rho}(\mathcal{P})\right) \rightarrow F(X)
$$


is a crossed module, as defined in Whitehead (1949). We give a presentation for $\operatorname{star}_{1}\left(\operatorname{Sq}^{\rho}(\mathcal{P})\right)$, and use it to show that the crossed module is isomorphic to that usually associated to a group presentation, as in Brown and Huebschmann (1982). It then follows that the fundamental group $\pi_{1}\left(\operatorname{Sq}^{\rho}(\mathcal{P}), 1\right)$ can be interpreted as the kernel of a free presentation of the relation module of $\mathcal{P}$, and as in Cremanns and Otto (1996) we may link the module structure of $\pi_{1}\left(\operatorname{Sq}^{\rho}(\mathcal{P}), 1\right)$ to the homological finiteness condition $\mathrm{FP}_{3}$, and as in Dyer (1993) to Cockcroft properties of $\mathcal{P}$.

\section{Background notions and notation}

\subsection{Groupoids}

A groupoid $G$ is a small category in which every morphism is invertible. We consider a groupoid as an algebraic structure as in Higgins (1971): the elements are the morphisms, and composition is an associative partial binary operation. The set of vertices of $G$ is denoted $V(G)$, and for each vertex $x \in V(G)$ there exists an identity morphism $1_{x}$. An element $g \in G$ has domain $g \mathbf{d}$ and range $g \mathbf{r}$ in $V(G)$, with $g g^{-1}=1_{g \mathbf{d}}$ and $g^{-1} g=1_{g \mathbf{r}}$. For $e \in V(G)$ the star of $e$ in $G$ is the $\operatorname{set} \operatorname{star}_{e}(G)=\{g \in G: g \mathbf{d}=e\}$, and the local group at $e$ is the set $G(e)=\{g \in G: g \mathbf{d}=e=g \mathbf{r}\}$.

\subsection{Crossed modules}

Crossed modules will be the algebraic models of group presentations that we shall use in our formulation of the relation module and the module of identities for a group presentation. For a more detailed account of these topics, we refer to Brown and Huebschmann (1982).

A crossed module is a group homomorphism $\partial: T \rightarrow \Gamma$ together with an action of $\Gamma$ on $T$ (written $(t, g) \mapsto t^{g}$ ) such that $\partial$ is $\Gamma$-equivariant, so that for all $t \in T$ and $g \in \Gamma$ we have

$$
\left(t^{g}\right) \partial=g^{-1}(t \partial) g
$$

and such that for all $t, u \in T$, we have:

$$
t^{u \partial}=u^{-1} t u
$$

We shall say that $(T, \partial)$ is a crossed $\Gamma$-module.

Example 1 Examples of crossed modules include the following:

- any $\Gamma$-module $M$ with the trivial map $M \stackrel{0}{\rightarrow} \Gamma$,

- the inclusion of any normal subgroup $N \hookrightarrow \Gamma$,

- the map $T \rightarrow$ Aut $T$ that associates to $t \in T$ the inner automorphism of $T$ defined by $a \mapsto t^{-1} a t$,

- any surjection $T \rightarrow \Gamma$ with central kernel, where $\Gamma$ acts on $T$ by lifting and conjugation, 
- the boundary map $\pi_{2}(X, Y) \rightarrow \pi_{1}(Y)$ from the second relative homotopy group of a pair of spaces $(X, Y)$ with $Y \subseteq X$.

The last example motivated the introduction of crossed modules by Whitehead (1949).

Let $\partial: T \rightarrow \Gamma$ be a crossed module, and let $N$ be the image of $\partial$. The following properties are easy consequences of (1) and (2).

- $N$ is normal in $\Gamma$, and so if we set $G=\Gamma / N$ we get the short exact sequence of groups: $1 \rightarrow N \rightarrow \Gamma \rightarrow G \rightarrow 1$.

- ker $\partial \subseteq Z(T)$, the center of $T$, so ker $\partial$ is abelian.

- $\operatorname{ker} \partial$ is invariant under the $\Gamma$-action on $T$, and so is a $\Gamma$-module.

- $N$ acts trivially on $Z(T)$ and thus on ker $\partial$, hence ker $\partial$ inherits an action of $G$ to become a $G$-module.

- the abelianisation $T^{a b}$ of $T$ inherits the structure of a $G$-module.

Definition 1 Let $(T, \partial)$ and $\left(T^{\prime}, \partial^{\prime}\right)$ be crossed $\Gamma$-modules. A morphism $\phi:(T, \partial) \rightarrow$ $\left(T^{\prime}, \partial^{\prime}\right)$ is a group homomorphism $\phi: T \rightarrow T^{\prime}$ such that for $t \in T$, and $g \in \Gamma$, $\left(t^{g}\right) \phi=(t \phi)^{g}$ and $\phi \partial^{\prime}=\partial$.

\subsubsection{Free crossed modules}

Definition 2 Let $(T, \partial)$ be a crossed $\Gamma$-module, let $R$ be a set, and let $\rho: R \rightarrow T$ be a function. We say $(T, \partial)$ is a free crossed $\Gamma$-module with basis $\rho$ if for any crossed $\Gamma$-module $\left(T^{\prime}, \partial^{\prime}\right)$ and function $\sigma: R \rightarrow T^{\prime}$ such that $\sigma \partial^{\prime}=\rho \partial$, that is, such that the square

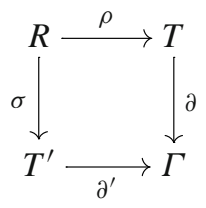

commutes, then there exists a unique morphism of crossed modules $\phi:(T, \partial) \rightarrow$ $\left(T^{\prime}, \partial^{\prime}\right)$ such that $\rho \phi=\sigma$, that is,

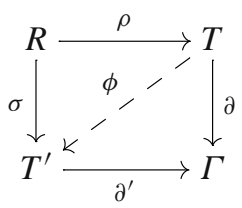

also commutes. We may also choose to emphasise $\omega=\rho \partial: R \rightarrow \Gamma$ by saying that a free crossed module $(T, \partial)$ with basis $\rho$ is a free crossed module on $\omega$.

The construction of free crossed modules is due to Whitehead (1949), and is also discussed by Brown and Huebschmann (1982). 
Proposition 1 (Whitehead 1949) Let $\Gamma$ be a group, $R$ a set, and $\omega: R \rightarrow \Gamma a$ function. Then a free crossed $\Gamma$-module on $\omega$ exists and is unique up to isomorphism.

Proof We sketch the construction, following (Brown and Huebschmann 1982, Proposition 5). Let $F$ be the free group on the basis $R \times \Gamma$. Then $\Gamma$ acts on $F$ by right multiplication of basis elements: for $r \in R$ and $u, v \in \Gamma$ we have $(r, u)^{v}=(r, u v)$. We map $(r, u) \mapsto u^{-1}(r \omega) u$ and this induces a group homomorphism $\delta: F \rightarrow \Gamma$. The subgroup $P$ of $F$ generated by all elements of the form

$$
(r, u)^{-1}(s, v)^{-1}(r, u)\left(s, v u^{-1}(r \omega) u\right)
$$

with $r, s \in R$ and $u, v \in \Gamma$ is normal in $F$, invariant under the $\Gamma$-action, and contained in $\operatorname{ker} \delta$. It follows that $\delta$ induces $\partial: F / P \rightarrow \Gamma$, and this is a free crossed $\Gamma$-module on $\omega$. Uniqueness up to isomorphism follows from the usual universal argument.

Whitehead also observed the following:

Proposition 2 (Whitehead 1949, page 457) Let $(C, \partial)$ be the free crossed $\Gamma$-module with basis $\rho$, and set $Q=$ coker $\partial$. Then $C^{a b}$ is a free $Q$-module on the image of the composition $\bar{\rho}: R \stackrel{\rho}{\rightarrow} C \rightarrow C^{a b}$.

\subsubsection{Crossed modules from group presentations}

A group presentation $\mathcal{P}=\langle X: \mathcal{R}\rangle$ of a group $G$, consists of a set of generators $X$, and a set of relations $\mathcal{R} \subseteq\left(X \cup X^{-1}\right)^{*} \times\left(X \cup X^{-1}\right)^{*}$. We set $A=X \cup X^{-1}$, we let $\rho: A^{*} \rightarrow F(X)$ be the canonical map, and we define $\hat{\rho}: \mathcal{R} \rightarrow F(X)$ by $(\ell, r) \hat{\rho}=\left(\ell^{-1} r\right) \rho$. We let $R$ be the image of $\hat{\rho}$ in $F(X)$, and define $N=\langle\langle R\rangle$ to be the normal closure of $R$ in $F$, so that a typical element of $N$ has the form

$$
u_{1}^{-1}\left(r_{1} \hat{\rho}\right)^{\varepsilon_{1}} u_{1} \cdots u_{k}^{-1}\left(r_{k} \hat{\rho}\right)^{\varepsilon_{k}} u_{k},
$$

where, for $1 \leq j \leq k$, we have $u_{j} \in F, r_{j} \in \mathcal{R}$, and $\varepsilon_{j}= \pm 1$. Then $G$ is the quotient group $F(X) / N$, and we have a canonical presentation map $\theta: F(X) \rightarrow G$.

We now let $(C(\mathcal{P}), \partial)$ be the free crossed $F(X)$-module on the function $\hat{\rho}: \mathcal{R} \rightarrow$ $F(X)$. An element of $C=C(\mathcal{P})$ is represented by a product

$$
\left(r_{1}, w_{1}\right)^{\varepsilon_{1}} \cdots\left(r_{k}, w_{k}\right)^{\varepsilon_{k}}
$$

where $r_{j} \in \mathcal{R}, w_{j} \in F(X)$ and $\varepsilon_{k}= \pm 1$. A typical Peiffer element (trivial in $C$ ) has the form

$$
(r, u)^{-1}(s, v)^{-1}(r, u)\left(s, v u^{-1}(r \hat{\rho}) u\right) .
$$

For $(r, w) \in C$ we have $\partial:(r, w) \mapsto w^{-1}(r \hat{\rho}) w$, and the image of $\partial$ is $N$. We denote ker $\partial$ by $\pi=\pi(\mathcal{P})$. We therefore have short exact sequences of groups

$$
1 \rightarrow N \rightarrow F(X) \rightarrow G \rightarrow 1
$$


and

$$
0 \rightarrow \pi(\mathcal{P}) \rightarrow C(\mathcal{P}) \rightarrow N \rightarrow 1,
$$

with $\pi$ central in $C$ and a $G$-module.

Proposition 3 (Brown and Huebschmann 1982, Corollary to Proposition 7) The free crossed module $C$ is isomorphic as a group to $\pi \times N$. Its abelianisation $C^{a b}$ is a free $G$-module, and the induced map $\pi \rightarrow C^{a b}$ is injective, so that we have a short exact sequence of $G$-modules.

$$
0 \rightarrow \pi \rightarrow C^{a b} \rightarrow N^{a b} \rightarrow 0
$$

Proof The group $N$ is free since it is a subgroup of $F(X)$, and so (4) splits. Since $\pi$ is central in $C$ we have $C \cong \pi \times N$. It follows that $[C, C] \cong\{0\} \times[N, N]$ and so $\pi \rightarrow C^{a b}$ is injective. $C^{a b}$ is free by Proposition 2 .

In the sequence (5), the $G$-module $N^{a b}$ is the relation module of the presentation $\mathcal{P}$, and the $G$-module $\pi$ is the module of identities. The sequence (5) then gives a free presentation of the relation module.

\section{Regular groupoids}

We now introduce some additional structure on a groupoid. This idea originates in Brown and Gilbert (1989), and was further developed in Gilbert (1998) and Brown (2010). Brown uses the terminology whiskered groupoid for what Gilbert had called a semiregular groupoid. We shall use the semiregular terminology, and will discuss in detail the special case of regular groupoids.

Definition 3 Let $G$ be a groupoid, with vertex set $V(G)$ and domain and range maps $\mathbf{d}, \mathbf{r}: G \rightarrow V(G)$. Then $G$ is semiregular if

- $V(G)$ is a monoid, with identity $e \in V(G)$,

- there are left and right actions of $V(G)$ on $G$, denoted $x \triangleright \alpha, \alpha \triangleleft x$, which for all $x, y \in V(G)$ and $\alpha, \beta \in G$ satisfy:

(a) $(x y) \triangleright \alpha=x \triangleright(y \triangleright \alpha) ; \alpha \triangleleft(x y)=(\alpha \triangleleft x) \triangleleft y$; $(x \triangleright \alpha) \triangleleft y=x \triangleright(\alpha \triangleleft y)$,

(b) $e \triangleright \alpha=\alpha=\alpha \triangleleft e$,

(c) $(x \triangleright \alpha) \mathbf{d}=x(\alpha \mathbf{d}) ;(\alpha \triangleleft x) \mathbf{d}=(\alpha \mathbf{d}) x ;(x \triangleright \alpha) \mathbf{r}=x(\alpha \mathbf{r}) ;(\alpha \triangleleft x) \mathbf{r}=(\alpha \mathbf{r}) x$,

(d) $x \triangleright(\alpha \circ \beta)=(x \triangleright \alpha) \circ(x \triangleright \beta) ;(\alpha \circ \beta) \triangleleft x=(\alpha \triangleleft x) \circ(\beta \triangleleft x)$, whenever $\alpha \circ \beta$ is defined,

(e) $x \triangleright 1_{y}=1_{x y}=1_{x} \triangleleft y$.

A semiregular groupoid $G$ is a regular groupoid if $V(G)$ is a group.

Our first result collates some simple facts from (Gilbert 1998, section 1).

Proposition 4 (a) Let $G$ be a semiregular groupoid. Then there are two everywhere defined binary operations on $G$ given by:

$$
\alpha * \beta=(\alpha \triangleleft \beta \mathbf{d}) \circ(\alpha \mathbf{r} \triangleright \beta)
$$




$$
\alpha \circledast \beta=(\alpha \mathbf{d} \triangleright \beta) \circ(\alpha \triangleleft \beta \mathbf{r}) .
$$

Each of the binary operations $*$ and $\circledast$ make $G$ into a monoid, with identity $1_{e}$.

(b) The binary operation $*$ and the monoid structure on $V(G)$ make the semiregular groupoid $G$ into a strict monoidal groupoid if and only if the operations $*$ and $\circledast$ on $G$ coincide.

(c) Let $G$ be a regular groupoid. Then each of the two binary operations $*$ and $\circledast$ given in Proposition 4 make $G$ into a group, with identity $1_{e}$.

(d) Let $G$ be a regular groupoid. Then $\mathbf{r}:(G, *) \rightarrow V(G)$ is a group homomorphism, and $\operatorname{star}_{e}(G)$ is a subgroup of $(G, *)$.

Proof We remark only on the proof of (c), since it is mis-stated in Gilbert (1998). The inverse of $\alpha$ with respect to $*$ is

$$
\alpha^{*}=\alpha \mathbf{r}^{-1} \triangleright \alpha^{\circ} \triangleleft \alpha \mathbf{d}^{-1}
$$

and with respect to $\circledast$ is

$$
\alpha^{\circledast}=\alpha \mathbf{d}^{-1} \triangleright \alpha^{\circ} \triangleleft \alpha \mathbf{r}^{-1}
$$

where $^{\circ}$ is the inverse of $\alpha$ with respect to the groupoid operation, and ${ }^{-1}$ is the inverse in the group $V(G)$.

Definition 4 In view of part (c) of Proposition 4, we say that a semiregular groupoid is monoidal if the operations $*$ and $\circledast$ coincide. [Brown (2010) calls such semiregular groupoids commutative.]

Still following (Gilbert 1998, section 1), we state the connection between regular groupoids and crossed modules.

Proposition 5 In a regular groupoid $G$, the group $(G, *)$ admits a group action of $V(G)$ by automorphisms, defined for $\alpha \in G$ and $q \in V(G)$ by $\alpha^{q}=q^{-1} \triangleright \alpha \triangleleft q$. Then $\mathbf{r}: \operatorname{star}_{e}(G) \rightarrow V(G)$ is a crossed module if and only if $G$ is monoidal.

\section{The Squier complex of a group presentation}

Let $\mathcal{P}=\langle X: \mathcal{R}\rangle$ be a group presentation. Recall from Sect. 1.2.2 that relations $(l, r) \in \mathcal{R}$ may involve words in $\left(X \cup X^{-1}\right)^{*}$ that are not freely reduced. However, to reduce notational clutter, we shall suppress mention of the free reduction map $\rho:\left(X \cup X^{-1}\right)^{*} \rightarrow F(X)$ in what follows. Hence if $p, q \in F(X)$ and $(l, r) \in \mathcal{R}$, we shall write $\operatorname{prq}$ for $p(r \rho) q$, and so on. The product prq is then interpreted as a product in $F(X)$, but need not of course be reduced as written.

Example 2 If $p=a b a^{-1}$ and $q=c^{-1} d c$ in $F(X)$, and $r=a c c^{-1} c \in\left(X \cup X^{-1}\right)^{*}$, then

$$
\operatorname{prq}=a b a^{-1} a c c^{-1} c c^{-1} d c=a b d c \in F(X)
$$


Definition 5 The reduced Squier complex $\operatorname{Sq}^{\rho}(\mathcal{P})$ is the 2-complex defined as follows:

- the vertex set of $\operatorname{Sq}^{\rho}(\mathcal{P})$ is the free group $F(X)$ on $X$,

- the edge set of $\operatorname{Sq}^{\rho}(\mathcal{P})$ consists of all 3-tuples $(p, l, r, q)$ with $p, q \in F(X)$ and $(l, r) \in \mathcal{R}$. Such an edge will start at $p l q$ and end at $p r q$, so each edge corresponds to the application of a relation in $F(X)$.

- the 2-cells correspond to applications of non-overlapping relations, and so a 2-cell is attached along every edge path of the form:

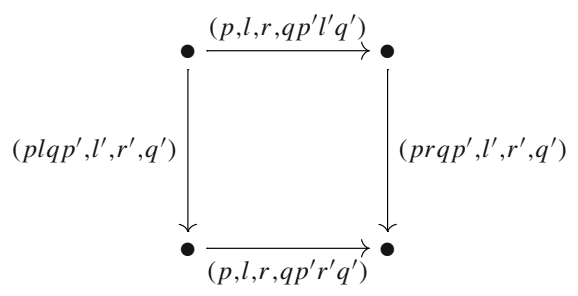

The edge paths

$$
\left(p, l, r, q p^{\prime} l^{\prime} q^{\prime}\right)\left(p r q p^{\prime}, l^{\prime}, r^{\prime}, q^{\prime}\right)
$$

and

$$
\left(p l q p^{\prime}, l^{\prime}, r^{\prime}, q^{\prime}\right)\left(p, l, r, q p^{\prime} r^{\prime} q^{\prime}\right)
$$

will therefore be homotopic in $\operatorname{Sq}^{\rho}(\mathcal{P})$.

Lemma 1 The fundamental groupoid $\pi\left(\operatorname{Sq}^{\rho}(\mathcal{P}), F(X)\right)$ of the Squier complex $\operatorname{Sq}^{\rho}(\mathcal{P})$ of a group presentation $\mathcal{P}$ is a regular groupoid.

Proof The vertex set of $\pi=\pi\left(\mathrm{Sq}^{\rho}(\mathcal{P}), F(X)\right)$ is the group $F(X)$. We need to define left and right actions of $F(X)$ on homotopy classes of paths in $\operatorname{Sq}^{\rho}(\mathcal{P})$. We first define such actions for single edges. Let $u, v \in F(X)$ and suppose that $(p, l, r, q)$ is an edge in $\operatorname{Sq}^{\rho}(\mathcal{P})$. We define

$$
\begin{aligned}
u \triangleright(p, l, r, q) & =(u p, l, r, q) \\
(p, l, r, q) \triangleleft v & =(p, l, r, q v) .
\end{aligned}
$$

It is then clear that these actions can be extended to edge-paths in $\operatorname{Sq}^{\rho}(\mathcal{P})$, and induce actions of $F(X)$ on homotopy classes of paths.

In what follows it will be convenient to work directly with edge paths in $\operatorname{Sq}^{\rho}(\mathcal{P})$, even though these are to be interpreted as representatives of homotopy classes in the fundamental groupoid $\pi\left(\mathrm{Sq}^{\rho}(\mathcal{P}), F(X)\right)$. In particular, we shall apply the operations $*$ and $\circledast$ directly to edge paths.

Theorem 1 The regular groupoid $\pi(S q(\mathcal{P}), F(X))$ is monoidal. 
Proof Recall that

$$
\begin{aligned}
\alpha * \beta & =(\alpha \triangleleft \beta \mathbf{d}) \circ(\alpha \mathbf{r} \triangleright \beta) \\
\alpha \circledast \beta & =(\alpha \mathbf{d} \triangleright \beta) \circ(\alpha \triangleleft \beta \mathbf{r}) .
\end{aligned}
$$

First we consider single-edge paths $\alpha=(p, l, r, q)$ and $\beta=\left(p^{\prime}, l^{\prime}, r^{\prime}, q^{\prime}\right)$. Then

$$
\begin{aligned}
\alpha * \beta & =\left(p, l, r, q p^{\prime} l^{\prime} q^{\prime}\right) \circ\left(p r q p^{\prime}, l^{\prime}, r^{\prime}, q^{\prime}\right) \\
\alpha \circledast \beta & =\left(p l q p^{\prime}, l^{\prime}, r^{\prime}, q^{\prime}\right) \circ\left(p, l, r, q p^{\prime} r^{\prime} q^{\prime}\right) .
\end{aligned}
$$

These paths comprise the boundary of a 2-cell in $\operatorname{Sq}^{\rho}(\mathcal{P})$ and are thus homotopic: hence $\alpha * \beta=\alpha \circledast \beta$.

Now consider edge paths $\alpha=\alpha_{1} \circ \alpha_{2} \circ \cdots \circ \alpha_{k}$ and $\beta=\beta_{1} \circ \beta_{2} \circ \cdots \circ \beta_{m}$ and with each $\alpha_{i}, \beta_{j}$ single edges. We set $m=1$ and $k>1$ : then we may assume that if $\beta$ is the single edge $\beta_{1}$ then

$$
\left(\alpha_{1} \circ \cdots \circ \alpha_{k-1}\right) * \beta_{1}=\left(\alpha_{1} \circ \cdots \circ \alpha_{k-1}\right) \circledast \beta_{1} .
$$

We then have

$$
\begin{aligned}
\alpha * \beta_{1} & =\left(\alpha \triangleleft \beta_{1} \mathbf{d}\right) \circ\left(\alpha_{k} \mathbf{r} \triangleright \beta_{1}\right) \\
& =\left(\alpha_{1} \triangleleft \beta_{1} \mathbf{d}\right) \circ\left(\alpha_{2} \triangleleft \beta_{1} \mathbf{d}\right) \circ \cdots \circ\left(\alpha_{k} \triangleleft \beta_{1} \mathbf{d}\right) \circ\left(\alpha_{k} \mathbf{r} \triangleright \beta_{1}\right) \\
& =\left(\alpha_{1} \triangleleft \beta_{1} \mathbf{d}\right) \circ \cdots \circ\left(\alpha_{k-1} \triangleleft \beta_{1} \mathbf{d}\right) \circ\left(\alpha_{k} * \beta_{1}\right) \\
& =\left(\alpha_{1} \triangleleft \beta_{1} \mathbf{d}\right) \circ \cdots \circ\left(\alpha_{k-1} \triangleleft \beta_{1} \mathbf{d}\right) \circ\left(\alpha_{k} \circledast \beta_{1}\right) \\
& =\left(\alpha_{1} \triangleleft \beta_{1} \mathbf{d}\right) \circ \cdots \circ\left(\alpha_{k-1} \triangleleft \beta_{1} \mathbf{d}\right) \circ\left(\alpha_{k} \mathbf{d} \triangleright \beta_{1}\right) \circ\left(\alpha_{k} \triangleleft \beta_{1} \mathbf{r}\right) \\
& =\left(\alpha_{1} \triangleleft \beta_{1} \mathbf{d}\right) \circ \cdots \circ\left(\alpha_{k-1} \triangleleft \beta_{1} \mathbf{d}\right) \circ\left(\alpha_{k-1} \mathbf{r} \triangleright \beta_{1}\right) \circ\left(\alpha_{k} \triangleleft \beta_{1} \mathbf{r}\right) \\
& =\left(\left(\alpha_{1} \circ \cdots \circ \alpha_{k-1}\right) * \beta_{1}\right) \circ\left(\alpha_{k} \triangleleft \beta_{1} \mathbf{r}\right) \\
& =\left(\left(\alpha_{1} \circ \cdots \circ \alpha_{k-1}\right) \circledast \beta_{1}\right) \circ\left(\alpha_{k} \triangleleft \beta_{1} \mathbf{r}\right) \\
& =\left(\alpha_{1} \mathbf{d} \triangleright \beta_{1}\right) \circ\left(\alpha_{1} \triangleleft \beta_{1} \mathbf{r}\right) \circ \cdots \circ\left(\alpha_{k-1} \triangleleft \beta_{1} \mathbf{r}\right) \circ\left(\alpha_{k} \triangleleft \beta_{1} \mathbf{r}\right) \\
& =\alpha \circledast \beta_{1}
\end{aligned}
$$

So by induction on $k$, we have $\alpha * \beta=\alpha \circledast \beta$, whenever $m=1$. Now for $m>1$ we assume inductively that, for any edge path $\alpha$,

$$
\alpha *\left(\beta_{1} \circ \cdots \circ \beta_{m-1}\right)=\alpha \circledast\left(\beta_{1} \circ \cdots \circ \beta_{m-1}\right) .
$$

Then

$$
\begin{aligned}
\alpha * \beta & =\left(\alpha \triangleleft \beta_{1} \mathbf{d}\right) \circ\left(\alpha_{n} \mathbf{r} \triangleright \beta\right) \\
& =\left(\left(\alpha_{1} \circ \cdots \circ \alpha_{n}\right) \triangleleft \beta_{1} \mathbf{d}\right) \circ\left(\alpha_{n} \mathbf{r} \triangleright\left(\beta_{1} \circ \cdots \circ \beta_{m-1}\right)\right) \circ\left(\alpha_{n} \mathbf{r} \triangleright \beta_{j}\right) \\
& =\left(\alpha *\left(\beta_{1} \circ \cdots \circ \beta_{m-1}\right)\right) \circ\left(\alpha_{n} \mathbf{r} \triangleright \beta_{m}\right) \\
& =\left(\alpha \circledast\left(\beta_{1} \circ \cdots \circ \beta_{m-1}\right)\right) \circ\left(\alpha_{n} \mathbf{r} \triangleright \beta_{m}\right)
\end{aligned}
$$




$$
\begin{aligned}
& =\left(\alpha_{1} \mathbf{d} \triangleright\left(\beta_{1} \circ \cdots \circ \beta_{m-1}\right)\right) \circ\left(\alpha \triangleleft \beta_{m-1} \mathbf{r}\right) \circ\left(\alpha_{n} \mathbf{r} \triangleright \beta_{j}\right) \\
& =\left(\alpha_{1} \mathbf{d} \triangleright\left(\beta_{1} \circ \cdots \circ \beta_{m-1}\right)\right) \circ\left(\alpha \triangleleft \beta_{m} \mathbf{d}\right) \circ\left(\alpha_{n} \mathbf{r} \triangleright \beta_{j}\right) \\
& =\left(\alpha_{1} \mathbf{d} \triangleright\left(\beta_{1} \circ \cdots \circ \beta_{m-1}\right)\right) \circ\left(\alpha * \beta_{m}\right) \\
& =\left(\alpha_{1} \mathbf{d} \triangleright\left(\beta_{1} \circ \cdots \circ \beta_{m-1}\right)\right) \circ\left(\alpha \circledast \beta_{m}\right) \\
& =\left(\alpha_{1} \mathbf{d} \triangleright\left(\beta_{1} \circ \cdots \circ \beta_{m-1}\right)\right) \circ\left(\alpha_{1} \mathbf{d} \triangleright \beta_{m}\right) \circ\left(\alpha \triangleleft \beta_{m} \mathbf{r}\right) \\
& =(\alpha \mathbf{d} \triangleright \beta) \circ(\alpha \triangleright \beta \mathbf{r}) \\
& =\alpha \circledast \beta
\end{aligned}
$$

Thus by induction we have that $\alpha * \beta=\alpha \circledast \beta$, for all edge paths $\alpha, \beta$ in $\operatorname{Sq}^{\rho}(\mathcal{P})$.

From Proposition 5 we have:

Corollary 1 The subset $\operatorname{star}_{1}\left(\pi\left(\mathrm{Sq}^{\rho}(\mathcal{P}), F(X)\right)\right.$ of the fundamental groupoid of the Squier complex $\mathrm{Sq}^{\rho}(\mathcal{P})$ is a group under the binary operation $*$, and the restriction of the range map is a crossed module

$$
\mathbf{r}: \operatorname{star}_{1}\left(\pi\left(\mathrm{Sq}^{\rho}(\mathcal{P}), F(X)\right)\right) \rightarrow F(X)
$$

\subsection{The crossed module of a Squier complex}

Our aim is now to show that the crossed module in Corollary 1 is isomorphic to the free crossed module $C \stackrel{\partial}{\rightarrow} F(X)$ derived from the presentation $\mathcal{P}$, as in Sect. 1.2.2. Furthering our blurring of the distinction between an edge path and its homotopy class in the fundamental groupoid, we shall abbreviate the group $\operatorname{star}_{1}\left(\pi\left(\operatorname{Sq}^{\rho}(\mathcal{P}), F(X)\right)\right)$ as $\operatorname{star}_{1}\left(\operatorname{Sq}^{\rho}(\mathcal{P})\right)$. We denote by $S_{1}$ the set of all edges $e \in \operatorname{Sq}^{\rho}(\mathcal{P})$ with $e \mathbf{d}=1$, that is

$$
\begin{aligned}
S_{1} & =\{(p, l, r, q): p, q \in F(X),(l, r) \in \mathcal{R}, p l q=1\} \\
& =\left\{\left(q^{-1} l^{-1}, l, r, q\right): q \in F(X),(l, r) \in \mathcal{R}\right\} .
\end{aligned}
$$

The condition that $p l q=1$ means ( if we momentarily undo our notational convention of supressing the map $\rho)$ that $p(l \rho) q$ freely reduces to the empty word. We shall denote the edge $\left(q^{-1} l^{-1}, l, r, q\right)$ by $\lambda_{l, r, q}$.

Let $e=(p, l, r, q)$ be an edge of $\mathrm{Sq}^{\rho}(\mathcal{P})$ in the connected component of $1 \in F(X)$, and define

$$
e \lambda=(e \mathbf{d})^{-1} \triangleright e=\lambda_{l, r, q} \in S_{1} .
$$

Proposition 6 Let $\alpha$ be an edge path in $\operatorname{star}_{1}\left(\mathrm{Sq}^{\rho}(\mathcal{P})\right)$. Then $\alpha$ is equal to a $*$-product of single edges in $S_{1}$. Thus the group $\left(\operatorname{star}_{1}\left(\operatorname{Sq}^{\rho}(\mathcal{P})\right)\right.$, *) is generated by $S_{1}$

Proof The claim is trivial for edge paths $\alpha$ of length 1, so now suppose that

$$
\alpha=\alpha_{1} \circ \alpha_{2} \circ \cdots \circ \alpha_{n}
$$


for some $n>1$, with each $\alpha_{i}$ a single edge. Set $\lambda_{i}=\alpha_{i} \lambda=\left(\alpha_{i} \mathbf{d}\right)^{-1} \triangleright \alpha_{i}$. Then $\lambda_{i} \in S_{1}$, and $\alpha_{1}=\lambda_{1}$. We now assume inductively that

$$
\alpha_{1} \circ \alpha_{2} \circ \cdots \circ \alpha_{n-1}=\lambda_{1} * \lambda_{2} * \cdots * \lambda_{n-1} .
$$

Then

$$
\begin{aligned}
\alpha & =\left(\alpha_{1} \circ \cdots \circ \alpha_{n-1}\right) \circ \alpha_{n} \\
& =\left(\alpha_{1} \circ \cdots \circ \alpha_{n-1}\right) \circ\left(\alpha_{n} \mathbf{d} \triangleright \lambda_{n}\right) \\
& =\left(\alpha_{1} \circ \cdots \circ \alpha_{n-1}\right) \circ\left(\alpha_{n-1} \mathbf{r} \triangleright \lambda_{n}\right) \\
& =\left(\alpha_{1} \circ \cdots \circ \alpha_{n-1}\right) * \lambda_{n} \\
& =\lambda_{1} * \lambda_{2} * \cdots * \lambda_{n-1} * \lambda_{n} .
\end{aligned}
$$

Therefore $\alpha=\lambda_{1} * \cdots * \lambda_{n}$.

Definition 6 We denote the product $\lambda_{1} * \cdots * \lambda_{n}$ used to represent $\alpha \in \operatorname{star}_{1}\left(\operatorname{Sq}^{\rho}(\mathcal{P})\right)$ in Proposition 6 by $\alpha \lambda$.

Lemma 2 Suppose that $\alpha \circ \beta \in \operatorname{star}_{1}\left(\operatorname{Sq}^{\rho}(\mathcal{P})\right)$. Then $(\alpha \circ \beta) \lambda=\alpha \lambda * \beta \lambda$.

We now want to understand the effect of homotopy of edge paths in $\operatorname{Sq}^{\rho}(\mathcal{P})$ on the $*-$ products defined in Proposition 6. We first consider a 1-homotopy, that is, the insertion of deletion of a pair of inverse edges. Let $\xi=\rho \circ \sigma$ in $S q(\mathcal{P})$, with $\rho \in \operatorname{star}_{1}\left(\operatorname{Sq}^{\rho}(\mathcal{P})\right)$. Then consider the homotopic path $\xi^{\prime}=\rho \circ \alpha \circ \alpha^{\circ} \circ \sigma$, with $\alpha$ a single edge. Then

$$
\begin{aligned}
\xi^{\prime} \lambda & =\rho \lambda * \alpha \lambda *\left(\alpha^{\circ}\right) \lambda * \sigma \lambda \\
& =\rho \lambda *\left[(\alpha \mathbf{d})^{-1} \triangleright \alpha *\left(\alpha^{\circ} \mathbf{d}\right)^{-1} \triangleright \alpha^{\circ}\right] * \sigma \lambda \\
& =\rho \lambda *\left[(\alpha \mathbf{d})^{-1} \triangleright \alpha *(\alpha \mathbf{r})^{-1} \triangleright \alpha^{\circ}\right] * \sigma \lambda \\
& \left.=\rho \lambda *\left[(\alpha \mathbf{d})^{-1} \triangleright \alpha \triangleleft 1\right) \circ(\alpha \mathbf{d})^{-1} \alpha \mathbf{r} \triangleright\left((\alpha \mathbf{r})^{-1} \triangleright \alpha^{\circ}\right)\right] * \sigma \lambda \\
& \left.\left.=\rho \lambda *\left[(\alpha \mathbf{d})^{-1} \triangleright \alpha\right) \circ(\alpha \mathbf{d})^{-1} \triangleright \alpha^{\circ}\right)\right] * \sigma \lambda \\
& =\rho \lambda * \sigma \lambda=\xi \lambda .
\end{aligned}
$$

Therefore a 1-homotopy applied to an edge path $\xi$ does not change the $*$-product $\xi \lambda$.

Suppose that we have a 2 -cell

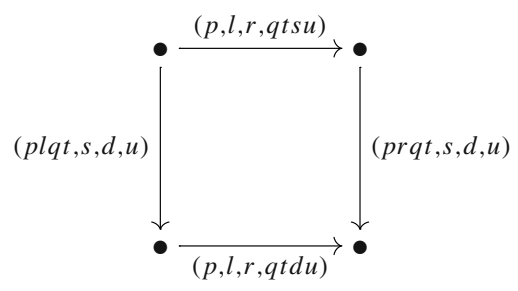


in the connected component of $1 \in F(X)$ in $\operatorname{Sq}^{\rho}(\mathcal{P})$, with

$$
\alpha=(p, l, r, q t s u), \beta=(p r q t, s, d, u), \gamma=(p l q t, s, d, u), \delta=(p, l, r, q t d u)
$$

This 2-cell gives a homotopy between $\alpha \circ \beta$ and $\gamma \circ \delta$, or equivalently tells us that in $\pi\left(\operatorname{Sq}^{\rho}(\mathcal{P})\right)$ we have

$$
(p, l, r, q) *(t, s, d, u)=(p, l, r, q) \circledast(t, s, d, u) .
$$

If this 2-cell is involved in a 2-homotopy between edge paths $\xi$ and $\xi^{\prime}$, we may assume using 1-homotopies where necessary, that we have $\xi=\rho \circ \alpha \circ \beta \circ \sigma$ and $\xi^{\prime}=\rho \circ \gamma \circ \delta \circ \sigma$, that is a configuration

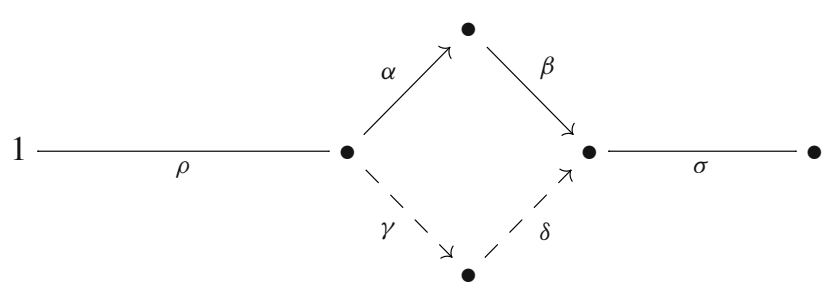

Then, using $\simeq$ to denote homotopy of edge paths in $\operatorname{Sq}^{\rho}(\mathcal{P})$, we have

$$
\begin{aligned}
\xi \lambda & =\rho \lambda * \alpha \lambda * \beta \lambda * \sigma \lambda \\
& =\rho \lambda *(\alpha \circ \beta) * \sigma \lambda \\
& \simeq \rho \lambda *(\gamma \circ \delta) * \sigma \lambda \\
& =\rho \lambda * \gamma \lambda * \delta \lambda * \sigma \lambda \\
& =\xi^{\prime} \lambda .
\end{aligned}
$$

The above considerations show that, for a given homotopy class in $\operatorname{star}_{1}\left(\operatorname{Sq}^{\rho}(\mathcal{P})\right)$, we may select a representative edge path $\xi$ in the form of its $*$-product $\xi \lambda$ and that this product will be unique up to changes induced by the 2-cells in $\operatorname{Sq}^{\rho}(\mathcal{P})$, which may modify the product as in Eq. (10) above. We can be more precise.

Proposition 7 Given $q \in F(X)$ and $(l, r) \in \mathcal{R}$, we set

$$
\lambda_{l, r, q}=\left(q^{-1} l^{-1}, l, r, q\right) \in S_{1}
$$

Then the following are a set of defining relations for the group $\left(\operatorname{star}_{1}\left(\mathrm{Sq}^{\rho}(\mathcal{P})\right), *\right)$ on the generating set $S_{1}$ :

$$
\lambda_{l, r, v s u} * \lambda_{s, d, u}=\lambda_{s, d, u} * \lambda_{l, r, v d u}
$$

where $(l, r),(s, d) \in \mathcal{R}$ and $u, v \in F(X)$. 


\section{Proof Since}

$$
\lambda_{l, r, v s u} * \lambda_{s, d, u}=\left(u^{-1} s^{-1} v^{-1} l^{-1}, l, r, v s u\right) \circ\left(u^{-1} s^{-1} v^{-1} l^{-1} r v, s, d, u\right)
$$

and

$$
\lambda_{s, d, u} * \lambda_{l, r, v d u}=\left(u^{-1}, s, d, u\right) \circ\left(u^{-1} v^{-1} l^{-1}, l, r, v d u\right),
$$

we see that the stated relations are true in $\left(\operatorname{star}_{1}\left(\operatorname{Sq}^{\rho}(\mathcal{P})\right), *\right)$ since they record the equality of the two paths around the sides of the 2-cell

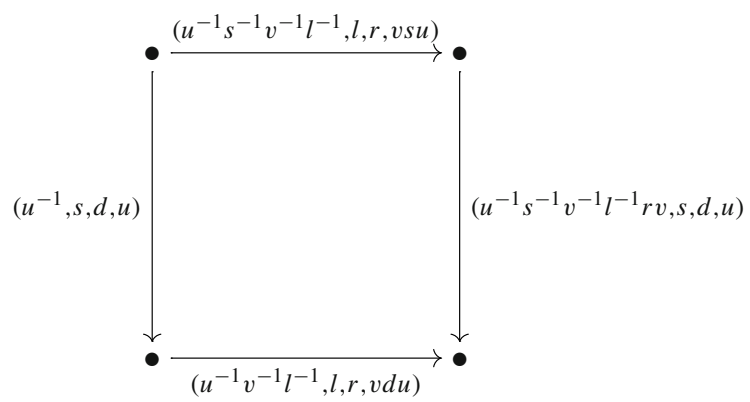

On the other hand, to accomplish the rewriting in (10), we need to identify the paths around the boundary of a general 2-cell as in (8) and, in the notation of (9), use the relation

$$
\alpha \lambda * \beta \lambda=\gamma \lambda * \delta \lambda
$$

Now

$$
\begin{aligned}
& \alpha \lambda=\left(u^{-1} s^{-1} t^{-1} q^{-1} l^{-1}, l, r, q t s u\right)=\left(l^{-1}, l, r, 1\right)^{q t s u}, \\
& \beta \lambda=\left(u^{-1} s^{-1}, s, d, u\right)=\left(s^{-1}, s, d, 1\right)^{u}=\gamma \lambda
\end{aligned}
$$

and

$$
\delta \lambda=\left(u^{-1} d^{-1} t^{-1} q^{-1} l^{-1}, l, r, q t d u\right)=\left(l^{-1}, l, r, 1\right)^{q t d u} .
$$

If we set $v=q t$ then

$$
\begin{aligned}
& \alpha \lambda=\left(u^{-1} s^{-1} v^{-1} l^{-1}, l, r, v s u\right)=\lambda_{l, r, v s u}, \\
& \beta \lambda=\lambda_{s, d, u}=\gamma \lambda
\end{aligned}
$$

and

$$
\delta \lambda=\left(u^{-1} d^{-1} v^{-1} l^{-1}, l, r, v d u\right)=\lambda_{l, r, v d u} .
$$


and the required relation is

$$
\lambda_{l, r, v s u} * \lambda_{s, d, u}=\lambda_{s, d, u} * \lambda_{l, r, v d u} .
$$

Theorem 2 The crossed $F(X)$-module $\operatorname{star}_{1}\left(\operatorname{Sq}^{\rho}(\mathcal{P})\right) \stackrel{\mathbf{r}}{\rightarrow} F(X)$ derived from the Squier complex $\operatorname{Sq}^{\rho}(\mathcal{P})$ of a group presentation $\mathcal{P}=\langle X: \mathcal{R}\rangle$, is isomorphic to the free crossed $F(X)$-module $C \stackrel{\partial}{\rightarrow} F$ derived from $\mathcal{P}$.

Proof Recall from Sect. 1.2.2 that the free crossed module $C \stackrel{\partial}{\rightarrow} F$ has basis function $v: \mathcal{R} \rightarrow C, v:(l, r) \mapsto(l, r, 1)$. We define $\bar{v}: \mathcal{R} \rightarrow \operatorname{star}_{1}\left(\operatorname{Sq}^{\rho}(\mathcal{P})\right)$ by $\bar{v}:$ $(l, r) \mapsto\left(l^{-1}, l, r, 1\right)$. Then $v \partial=\bar{v} \mathbf{r}$, and thus by freeness of $(C, \partial)$, we have a crossed module morphism $\phi: C \rightarrow \operatorname{star}_{1}\left(\operatorname{Sq}^{\rho}(\mathcal{P})\right)$, defined on generators by $(l, r, u) \mapsto$ $\left(u^{-1} l^{-1}, l, r, u\right)=\lambda_{l, r, u}$. We note that this is a bijection from the group generating set of $C$ to $S_{1}$.

To obtain an inverse to $\phi$, we therefore wish to map $\lambda_{l, r, u} \mapsto(l, r, u)$. This will be well-defined and a homomorphism if and only if the defining relations given in (11) in Proposition 7 are mapped to an equation that holds in the group $C$. Now the left-hand side of (11) maps to

$$
(l, r, v s u)(s, d, u)
$$

and the right-hand side to

$$
(s, d, u)(l, r, v d u) .
$$

and in the crossed $F(X)$-module $C$ we do indeed have

$$
(s, d, u)^{-1}(l, r, v s u)(s, d, u)=\left(l, r, v s u\left(u^{-1} s^{-1} d u\right)\right)=(l, r, v d u) .
$$

The kernel of the map $\mathbf{r}: \operatorname{star}_{1}\left(\mathrm{Sq}^{\rho}(\mathcal{P})\right) \rightarrow F(X)$ is the local group at $1 \in F(X)$ of the groupoid $\pi\left(\operatorname{Sq}^{\rho}(\mathcal{P}), F(X)\right)$, that is the fundamental group $\pi_{1}\left(\operatorname{Sq}^{\rho}(\mathcal{P}), 1\right)$. Then from Proposition 3 we obtain:

Proposition 8 Let $\mathcal{P}=\langle X: \mathcal{R}\rangle$ be a presentation of a group $G$ with presentation map $\theta: F(X) \rightarrow G$ and let $N=\operatorname{ker} \theta$, so that $N^{a b}$ is the relation module of $\mathcal{P}$. Then we have a short exact sequence of $G$-modules:

$$
0 \rightarrow \pi_{1}\left(\operatorname{Sq}^{\rho}(\mathcal{P}), 1\right) \rightarrow \bigoplus_{r \in \mathcal{R}} \mathbb{Z} G \rightarrow N^{a b} \rightarrow 0
$$

Example 3 Let $\mathcal{P}=\left\langle x: x x^{-1}=1\right\rangle$ presenting the infinite cyclic group $\langle x\rangle$. Then the relation modle is trivial, and (12) reduces to an isomorphism $\pi_{1}\left(\mathrm{Sq}^{\rho}(\mathcal{P}), 1\right) \cong \mathbb{Z}\langle x\rangle$. 
We can also see this from the construction of $\operatorname{Sq}^{\rho}(\mathcal{P})$. The $\operatorname{Squier}$ complex $\operatorname{Sq}^{\rho}(\mathcal{P})$ has vertex set $\langle x\rangle$ and each edge is a loop. The generating set $S_{1}$ in Proposition 6 is

$$
S_{1}=\left\{\left(x^{-q}, x x^{-1}, 1, x^{q}\right): q \in \mathbb{Z}\right\}
$$

and we write $\lambda_{q}=\left(x^{-q}, x x^{-1}, 1, x^{q}\right)$. By Proposition 7 we have a presentation for $\pi_{1}\left(\operatorname{Sq}^{\rho}(\mathcal{P}), 1\right)=\operatorname{star}_{1}\left(\operatorname{Sq}^{\rho}(\mathcal{P})\right)$ given by

$$
\pi_{1}\left(\operatorname{Sq}^{\rho}(\mathcal{P}), 1\right)=\left\langle\lambda_{q}(q \in \mathbb{Z}): \lambda_{p+q} * \lambda_{q}=\lambda_{q} * \lambda_{p+q}(p, q \in \mathbb{Z})\right\rangle
$$

and so $\pi_{1}\left(\operatorname{Sq}^{\rho}(\mathcal{P}), 1\right)$ is free abelian of countably infinite rank, and the $\langle x\rangle$-action is defined by $\lambda_{q}^{x}=\lambda_{q+1}$.

\subsection{Properties of $\pi_{1}\left(\mathrm{Sq}^{\rho}(\mathcal{P}), 1\right)$}

We show in two Corollaries of Proposition 8 how properties of the presentation $\mathcal{P}$ and the group $G$ are reflected in properties of the fundamental group of the reduced Squier complex. The illustrative examples that we give are drawn from Cremanns and Otto (1996) and Dyer (1993).

The first result was proved for the Squier complex of Squier et al. (1994) by Cremanns and Otto. We refer to Brown (1982, Chapter VIII) and to Cremanns and Otto (1996, section 4) for information on the condition $\mathrm{FP}_{3}$

Corollary 2 (Cremanns and Otto 1996, Theorem 4.10) Let $G$ be presented by the finite presentation $\mathcal{P}=\langle X: \mathcal{R}\rangle$. Then the following are equivalent.

(a) $\pi_{1}\left(\mathrm{Sq}^{\rho}(\mathcal{P}), 1\right)$ is a finitely generated $G$-module,

(b) $G$ is of type $\mathrm{FP}_{3}$.

Proof There is an exact sequence of $G$-modules (see Brown 1982, Proposition II.5.4),

$$
0 \rightarrow N^{a b} \rightarrow \bigoplus_{x \in X} \mathbb{Z} G \rightarrow \mathbb{Z} G \rightarrow \mathbb{Z} \rightarrow 0
$$

and if $\pi_{1}\left(\mathrm{Sq}^{\rho}(\mathcal{P}), 1\right)$ is a finitely generated as a $G$ module by a set $S$ this extends, using (12), to a partial free resolution of finite type

$$
\bigoplus_{S} \mathbb{Z} G \rightarrow \bigoplus_{\mathcal{R}} \mathbb{Z} G \rightarrow \bigoplus_{X} \mathbb{Z} G \rightarrow \mathbb{Z} G \rightarrow \mathbb{Z} \rightarrow 0
$$

which shows that $G$ has type $\mathrm{FP}_{3}$. Conversely, if $G$ has type $\mathrm{FP}_{3}$ then $\pi_{1}\left(\operatorname{Sq}^{\rho}(\mathcal{P}), 1\right)$ is the kernel (at dimension 2) in a partial free resolution of $\mathbb{Z}$ of finite type and so is finitely generated as a consequence of the generalized Schanuel Lemma, see Brown (1982, Proposition 4.3). 
The second result characterizes the Cockcroft properties of $\mathcal{P}$. Following Dyer (1993, Theorem 4.2) we make the following definition. Let $L$ be a subgroup of $G$, and apply the tensor product $-\otimes_{L} \mathbb{Z}$ to the sequence (12) to obtain the sequence

$$
\pi_{1}\left(\operatorname{Sq}^{\rho}(\mathcal{P}), 1\right) \otimes_{L} \mathbb{Z} \rightarrow \bigoplus_{r \in \mathcal{R}} \mathbb{Z}(G / L) \rightarrow N^{a b} \otimes_{L} \mathbb{Z} \rightarrow 0
$$

of abelian groups, where $G / L$ is the set of left cosets of $L$ in $G$. Then $\mathcal{P}$ is $L$-Cockcroft if the map

$$
\bigoplus_{r \in \mathcal{R}} \mathbb{Z}(G / L) \rightarrow N^{a b} \otimes_{L} \mathbb{Z}
$$

in (13) is an isomorphism. Immediately from (13) we obtain part of Dyer (1993, Theorem 4.2) in terms of $\pi_{1}\left(\mathrm{Sq}^{\rho}(\mathcal{P}), 1\right)$.

Corollary 3 (Dyer 1993, Theorem 4.2) Let $G$ be presented by the presentation $\mathcal{P}=$ $\langle X: \mathcal{R}\rangle$ and let $L$ be a subgroup of $G$. Then the following are equivalent:

(a) $\mathcal{P}$ is L-Cockcroft,

(b) the map $\pi_{1}\left(\mathrm{Sq}^{\rho}(\mathcal{P}), 1\right) \otimes_{L} \mathbb{Z} \rightarrow \bigoplus_{r \in \mathcal{R}} \mathbb{Z}(G / L)$ in (13) is the zero map.

Acknowledgements A version of these results is presented in the second author's $\mathrm{PhD}$ thesis at Heriot-Watt University, Edinburgh. The generous financial support of a PhD Scholarship from the Carnegie Trust for the Universities of Scotland is duly and gratefully acknowledged. We are grateful to the referee for the careful and scholarly scrutiny given to our paper.

Open Access This article is licensed under a Creative Commons Attribution 4.0 International License, which permits use, sharing, adaptation, distribution and reproduction in any medium or format, as long as you give appropriate credit to the original author(s) and the source, provide a link to the Creative Commons licence, and indicate if changes were made. The images or other third party material in this article are included in the article's Creative Commons licence, unless indicated otherwise in a credit line to the material. If material is not included in the article's Creative Commons licence and your intended use is not permitted by statutory regulation or exceeds the permitted use, you will need to obtain permission directly from the copyright holder. To view a copy of this licence, visit http://creativecommons.org/licenses/by/4.0/.

\section{References}

Anick, D.J.: On the homology of associative algebras. Trans. Am. Math. Soc. 296, 641-659 (1986)

Brown, K.S.: Cohomology of Groups. Graduate Texts in Math., vol. 87. Springer, New York (1982)

Brown, R., Huebschmann, J.: Identities among relations. In: Brown, R., Thickstun, T.L. (eds.) Low dimensional topology, London Math. Soc. Lect. Notes, vol. 48, pp. 153-202. Cambridge University Press, Cambridge

Brown, R., Gilbert, N.D.: Automorphism structures for crossed modules and algebraic models of 3-types. Proc. Lond. Math. Soc 59(3), 51-73 (1989)

Brown, R.: Possible connections between whiskered categories and groupoids, Leibniz algebras, automorphism structures and local-to-global questions. J. Homotopy Relat. Struct. 1(1), 1-13 (2010)

Cohen, D.E.: String rewriting - a survey for group theorists. In: Niblo, G.A., Roller, M.A. (eds.) Geometric group theory, London Math. Soc. Lect. Notes, vol. 181, pp. 37-47. Cambridge University Press, Cambridge (1993)

Cremanns, R., Otto, F.: For groups the property of having finite derivation type is equivalent to the homological finiteness condition $F P_{3}$. J. Symb. Comput. 22, 155-177 (1996) 
Dyer, M.N.: Crossed modules and $\Pi_{2}$ homotopy modules. In: Hog-Angeloni, C., et al. (eds) Twodimensional Homotopy and Combinatorial Group Theory, London Math. Soc. Lect. Notes, vol. 197, pp. 125-156. Cambridge University Press, Cambridge (1993)

Gilbert, N.D.: Monoid presentations and associated groupoids. Int. J. Algebra Comput. 8, 141-152 (1998)

Guba, V.S., Sapir, M.V.: Diagram groups. Memoirs Am. Math. Soc. 130, 1-117 (1997)

Guba, V.S., Sapir, M.V.: Diagram groups and directed 2-complexes: homotopy and homology. J. Pure Appl. Algebra 205, 1-47 (2006)

Higgins, P.J.: Notes on categories and groupoids. Van Nostrand Reinhold Math. Stud., vol. 32 (1971) (Reprinted electronically at) www.tac.mta.co/tac/reprints/articles/7/7tr7.pdf

Kilibarda, V.: On the algebra of semigroup diagrams. Int. J. Algebra Comput. 7, 313-338 (1997)

Otto, F., Kobayashi, Y.: Properties of monoids that are presented by finite convergent string-rewriting systems-a survey. In: Advances in algorithms, languages, and complexity, pp. 225-266. Kluwer Academic Publishers, Dordrecht (1997)

Pride, S.J.: Low-dimensional homotopy theory for monoids I. Int. J. Algebra Comput. 5, 631-649 (1995)

Pride, S.J.: Low-dimensional homotopy theory for monoids II: groups. Glasg. Math. J. 41, 1-11 (1999)

Squier, C.C.: Word problems and a homological finiteness condition for monoids. J. Pure Appl. Algebra 49, 201-216 (1987)

Squier, C.C., Otto, F., Kobayashi, Y.: A finiteness condition for rewriting systems. Theor. Comput. Sci. 131, 271-294 (1994)

Whitehead, J.H.C.: Combinatorial homotopy II. Bull. Am. Math. Soc. 55, 453-496 (1949)

Publisher's Note Springer Nature remains neutral with regard to jurisdictional claims in published maps and institutional affiliations. 